\title{
Serotonergic Afferents to the Rat Olfactory Bulb: II. Changes in Fiber Distribution During Development
}

\author{
John H. McLean ${ }^{1}$ and Michael T. Shipley ${ }^{1,2}$ \\ ${ }^{1}$ Department of Anatomy and Cell Biology, Division of Neurobiology, and ${ }^{2}$ Department of Neurosurgery, University of \\ Cincinnati College of Medicine, Cincinnati, Ohio 45267
}

The present studies have defined the developmental time course and distribution patterns of serotonergic fibers in the main olfactory bulb (MOB) using immunocytochemistry, anterograde tracing and image analysis. The results indicate that the deployment of serotonergic fibers to the main olfactory bulb is essentially a postnatal event in the rat. During the first $4 \mathrm{~d}$ after birth, 5-HT fibers infiltrate and begin to arborize in the MOB. The density of fibers in each layer is sparse during this period, but increases rapidly. By postnatal day 8 all layers are much more heavily innervated by $5-\mathrm{HT}$ fibers. The surge of fiber growth into all layers is rapid; the fibers arborize earlier at caudal than at rostral levels. This may be related to the increased metabolic activity that is reported to occur selectively in the caudal parts of the immature olfactory bulb.

After the second postnatal week, 5-HT fiber density increases much more gradually in all layers except the glomerular layer; in the glomerular layer, 5-HT fiber density continues to increase rapidly. It is also during this time that the olfactory bulb begins to grow substantially in volume. Bulb volume increases from the second week into adulthood (>60 d); during the same period, the density of 5-HT remains relatively constant in the infraglomerular layers. Thus, from the second week onward, the growth of 5-HT fibers appears to be closely linked to the increasing volumes of these layers. The density of 5-HT fibers in the glomerular layer, however, continues to increase from the second postnatal week. Thus, the density of fibers in the glomerular layer increases more than the increase in glomerular size, indicating that the glomerular 5-HT fibers are proliferating more than could be accounted for by simple glomerular expansion. In the adult, 5-HT fibers are 2-3 times denser in the glomerular than the infraglomerular layers (McLean and Shipley, 1987). This preferential innervation of glomeruli may be the result of a protracted period of arborization by glomerular versus infraglomerular fibers. This could be due to the prolonged focal release of a trophic factor by glomerular-associated neurons or to the earlier production of an inhibitory factor by infraglomerular neurons.

\footnotetext{
Received July 25, 1986; revised Mar. 27, 1987; accepted Apr. 1, 1987.

We thank E. Sieloff, M. Lazoff, B. Frydel, Y. Tsai, and K. Wilburn for technical assistance, and J. Fley and S. Harding for typing the manuscript. Many of the software programs for the Magiscan image analysis system were developed in this laboratory by J. Luna and P. Hug. This work was supported by Grants DAMD 17-86-C-6005 and NS-23348.

Correspondence should be addressed to Dr. Michael T. Shipley at the above address.

Copyright $(c) 1987$ Society for Neuroscience $0270-6474 / 87 / 103029-11 \$ 02.00 / 0$
}

The raphe serotonergic projection systems have been implicated in a number of functions, such as sleep (Jouvet, 1962), temperature regulation (Feldberg and Myers, 1964; Myers et al., 1969), hormone secretion (Mizuno et al., 1967), sensory response regulation (Srebro and Lorens, 1975), and analgesia (Tenen, 1968; Vogt, 1974; Basbaum and Fields, 1979). The variety of these suggested "general" functions undoubtedly reflects the widespread distribution of serotonin fibers in the CNS. It has recently been suggested that serotonin may also have trophic or regulatory functions during development. Lauder et al. (1982) hypothesized that serotonin release from fibers may have an epigenetic influence on proliferating neurons and glia. This proposal is based on studies in which serotonin depletion in developing brains resulted in delayed lamination of cerebral cortical fields that are innervated by serotonin fibers (Lauder et al., 1982). On the other hand, Lidov and Molliver (1982) presented immunocytochemical evidence that serotonin fibers do not innervate brain areas that are still in an immature, proliferative state. These investigators suggested, therefore, that the serotonin innervation is dependent on the maturity of target structure and further suggested that the relatively late arrival of the 5-HT fibers may be related more to the consolidation of cortical circuits than to stimulation of neuronal proliferation. It has also been reported that serotonin inhibits neurite outgrowth (Haydon et al., 1984). This suggests that serotonin might be able to regulate or influence the formation of cell-cell contacts or synaptic formation during development. To assess these and other potential developmental roles for 5-HT, it would be useful to study the development of 5-HT fibers in relation to the development of a relatively simple target system. Such a system is afforded by the olfactory bulb.

In the preceding paper (McLean and Shipley, 1987) we established that the main olfactory bulb (MOB) receives a heavy innervation from the dorsal and median raphe nuclei. Up to 1300 raphe neurons, the majority serotonergic, terminate in MOB. There is a particularly dense innervation of one layer, the glomerular layer, and a moderate (2-3 times less dense) innervation of the infraglomerular layers.

Because its major layers are already recognizable at birth, the olfactory bulb provides a distinctively laminated cortical model with which to study the relationship between the development of 5-HT innervation and the developmental status of the target structure. Another useful feature of this system is that its maturation is substantially postnatal, in contrast to the neocortex, where many equivalent processes are prenatal. Thus, the olfactory bulb and its connectional affiliates are relatively immature and are readily accessible to experimental manipulations at very 
early stages of development. The purpose of the present study was to characterize the development of serotonergic fibers in MOB as a step towards the assessment of possible role(s) of these fibers in regulating specific developmental events.

\section{Materials and Methods}

Perfusion. Rats aged 1, 2, 3, 4, 5, 8, 10, 11, 14, 16, 22, 28, 32, 37, 50, $60,64,73,83$, and $150 \mathrm{~d}$ were used in this study. The day of birth was considered postnatal day (PND) 1. The rats were deeply anesthetized with sodium pentobarbital and perfused transcardially with saline (room temperature) for $2 \mathrm{~min}$. The saline rinse was followed by perfusion with $4 \%$ paraformaldehyde and $0.05 \%$ glutaraldehyde in $0.1 \mathrm{~m}$ phosphate buffer ( $\mathrm{pH} \mathrm{7.4)}$ for $30 \mathrm{~min}(500 \mathrm{ml})$. In the course of this study, it became clear that optimum immunocytochemical staining was achieved when animals were perfused on ice with ice-cold fixatives. All morphometric analysis was done on cases processed in this way. Brains were removed from the skull, left in cold fixative for at least $1 \mathrm{hr}$, and placed in cold $20 \%$ sucrose in phosphate buffer overnight.

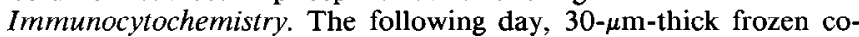
ronal or parasagittal sections of the olfactory bulb and rostral forebrain were cut into $0.1 \mathrm{M}$ PBS, $\mathrm{pH} 7.4$. Additional olfactory bulbs were sectioned and immediately placed onto subbed slides in a cryostat. These sections were processed for immunocytochemistry on the slides. Both free-floating and slide-mounted sections were processed for 5-HT immunocytochemistry following a protocol described in the previous paper (McLean and Shipley, 1987). Briefly, sections were (1) rinsed in $0.1 \mathrm{M}$ PBS, (2) incubated in primary antibody or without primary antibody in $0.1 \%$ Triton X-100, $2 \%$ normal goat serum (NGS) in PBS for 15-40 $\mathrm{hr}$ in the cold on a shaker, and then (3) rinsed twice for $10 \mathrm{~min}$ in PBS, (4) incubated in goat anti-rabbit biotinylated IgG (Vector Labs, Burlingame, CA) in the same diluent as in (1) for $1 \mathrm{hr}$ at room temperature, (5) rinsed twice for $10 \mathrm{~min}$ in PBS, and (6) incubated for $1 \mathrm{hr}$ in avidinbiotin-peroxidase complex (Vector Labs) in 0.1 м PBS. Procedures (3)(6) were sometimes repeated. After two $10 \mathrm{~min}$ rinses in PBS, sections were incubated in $0.05 \%$ diaminobenzidine dihydrochloride (DAB) with $0.01 \% \mathrm{H}_{2} \mathrm{O}_{2}$ in $0.1 \mathrm{M}$ phosphate buffer for $10-20 \mathrm{~min}$. Sections were then rinsed thoroughly in phosphate buffer and mounted on glass slides, rinsed in $\mathrm{H}_{2} \mathrm{O}$, dehydrated, and coverslipped. In some cases, the mounted sections were dipped for $1 \mathrm{~min}$ in $1 \% \mathrm{OsO}_{4}$ following lipid removal and dehydration in order to enhance DAB-stained profiles.

The primary antibody was 5-HT conjugated to bovine serum albumin (Immunonuclear) diluted 1:500-1:1000. Absorption controls on rat olfactory bulb sections were performed to determine the specificity of the 5-HT antibody. When a working dilution of the antibody was absorbed with an excess of the antigen complex, 5-HT-bovine serum albumin, no label was observed in olfactory bulb sections.

Anterograde tracing. A potential pitfall in inferring the distribution of developing raphe fibers using immunocytochemistry is that fibers may be present but may contain insufficient levels of 5-HT to be detected by immunocytochemistry. Therefore, we wished to determine whether the distribution of 5-HT-immunoreactive fibers matched the distribution of the afferent input from the raphe nuclei. To assess this, anterograde tracing studies were performed. In 2 PND 14 rats, $40 \mathrm{nl}$ of $1 \%$ WGA-HRP was injected stereotaxically into the dorsal and median raphe nuclei. Two days after the injections, the animals were deeply anesthetized and perfused transcardially according to the method of Mesulam (1978). Frozen sections, $40 \mu \mathrm{m}$ thick, were reacted for HRP histochemistry (Shipley, 1982). Sections were counterstained with neutral red and examined under dark-field polarized optics.

Image analysis. Transverse sections of the rat olfactory bulb were examined under bright- or dark-field microscope optics. A real-time video processor (Image $\Sigma$ Model 794, Hughes Aircraft Co.) was used to enhance the contrast of the labeled fibers, thus improving their detectability. The enhanced image was fed to an image-analysis system (Magiscan 2A; Joyce-Loebl). From the enhanced video image, fibers were identified and reconstructed in cytoarchitectonically defined olfactory bulb layers, using software developed in this laboratory. The imageanalysis system operates on a "live" digital image; images of $512 \times 512$ pixels $\times 6$ bits are displayed, but the Magiscan drives a motorized stage on the microscope so that the reconstructions are kept precisely in register with the image as the section is moved by the motorized stage. As a result, reconstruction of "live" images of $1024 \times 1024$ pixels and greater are generated. The Magiscan also controls the vertical position of the stage such that fibers can be imaged and reconstructed throughout the thickness of the section. To determine the length and density of serotonergic fibers in the developing MOB, the lengths of all fibers in each olfactory bulb layer were separately summed in standard regions of sections taken through the middle level of MOB. Fiber measurements were taken from midventral to dorsal regions on the lateral side of the olfactory bulb in 3-4 animals at each age. These measurements were used to calculate trends of fiber deployment during devclopment.

\section{Results}

The distribution of serotonergic fibers in the MOB of developing rats is detailed below. Because the distribution and density patterns at some ages are similar, data from these ages were grouped for purposes of description and analysis. The following descriptions are based on observations of midrostrocaudal levels of MOB unless otherwise stated, although all rostrocaudal levels were analyzed.

\section{PND 1-2}

The MOB is quite immature at birth. It is about $1-1.5 \mathrm{~mm}$ in diameter and roughly circular in coronal sections. As opposed to the adult olfactory bulb, which shows 6 distinct layers glomerular, external plexiform, mitral cell, internal plexiform, granule cell layers, and ependymal zone-the newborn rat's olfactory bulb has less distinct glomerular and internal plexiform layers. However, the external plexiform, mitral, and granule cell layers and ependymal zone are clearly distinguishable at this stage, so the major layers of the bulb can be specified.

5-HT-immunoreactive fibers are sparsely distributed in all layers at PND 1-2 (Figs. 1, 2A, 3). Some fibers run 200-300 $\mu \mathrm{m}$ along the glomerular layer in the plane of section. These fibers give off occasional collaterals within the glomerular layer or collaterals which extend into the external plexiform layer. In general, the fibers frequently cross laminar boundaries and are similar in size and morphology in all layers. They are approximately $0.30 \mu \mathrm{m}$ in diameter, with varicosities that average around $1 \mu \mathrm{m}$ in thickness. The varicosities are spaced $1-7 \mu \mathrm{m}$ apart. Frequently, the fibers end in swellings that are larger (1.6$1.8 \mu \mathrm{m})$ than the average varicosity. The appearance of these terminal swellings is suggestive of growth cones.

Caudal levels of the MOB have more 5-HT fibers than do rostral levels, suggesting that they are the first to be innervated by serotonergic fibers. The more caudally located accessory olfactory bulb, anterior olfactory nucleus (Fig. 1), and most parts of the cerebral cortex are much more densely innervated by serotonergic fibers at this early age than is the MOB.

\section{PND 4-6}

At this stage, the olfactory bulb has enlarged to $1.3-1.8 \mathrm{~mm}$ in diameter but is still essentially circular in cross section. The glomeruli are small but distinguishable, averaging $35-75 \mu \mathrm{m}$ in diameter. The external plexiform layer is only about $60 \mu \mathrm{m}$ thick, compared to $100-300 \mu \mathrm{m}$ in adults. At this early stage, the ependymal zone is still quite thick, approximately $200 \mu \mathrm{m}$ in radius. There is a marked overall increase in the density of 5-HT-immunoreactive fibers observed at all rostrocaudal levels of MOB as compared to the PND 1-2 stage (Fig. 4). Again, many fibers run parallel to the surface of the bulb, especially in the glomerular, deep external plexiform, and internal plexiform layers, while in the granule cell layer fibers are more randomly oriented. Occasionally, fibers end as enlarged varicosities, similar to the putative growth cones observed in olfactory bulbs of newborn rats. At this stage, fibers running circumferentially in 


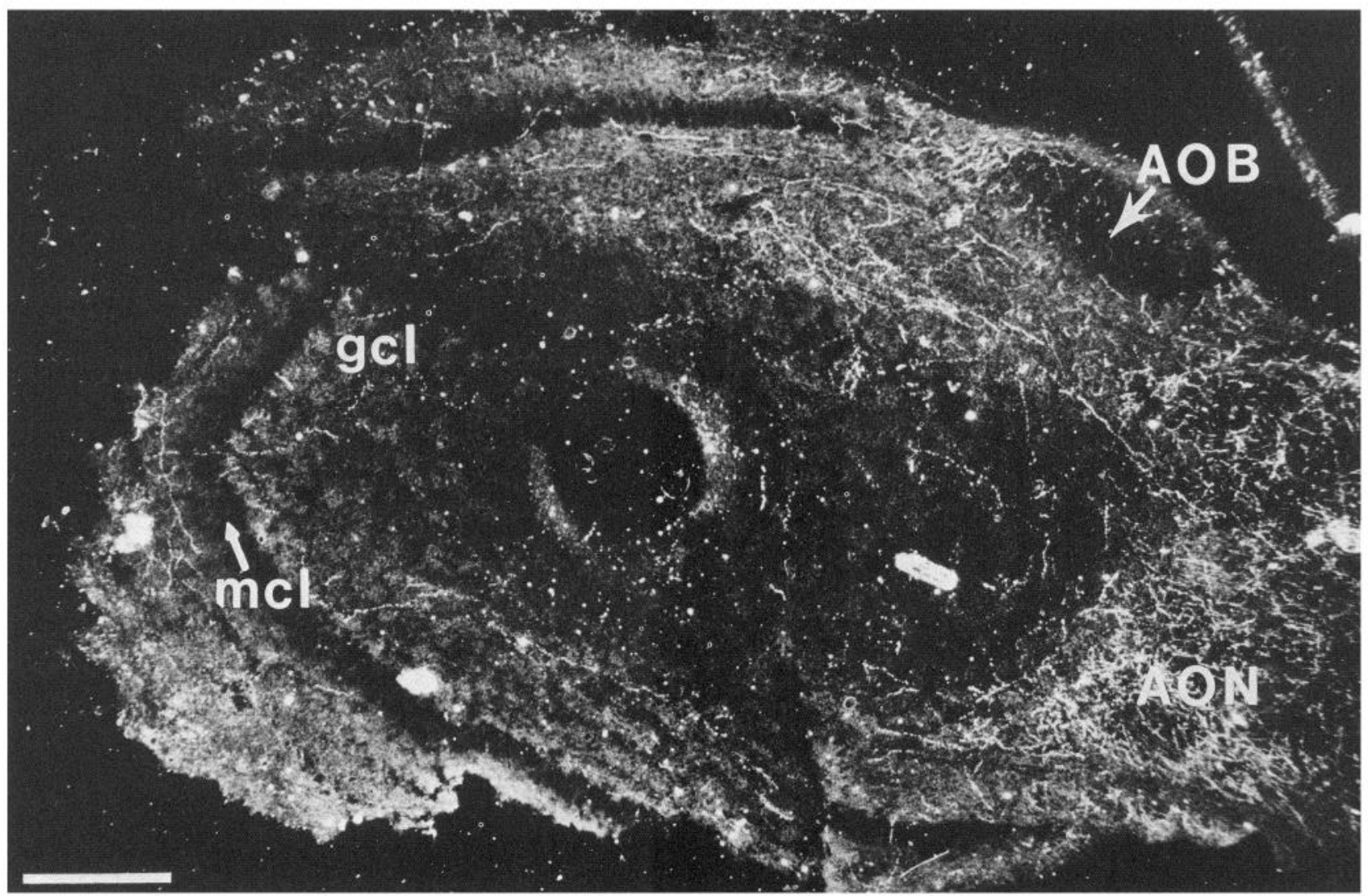

Figure 1. Dark-field photomicrograph of 5-HT-immunoreactive fibers in the olfactory bulb of a PND 1 rat (sagittal section). AOB, Accessory olfactory bulb; $\mathrm{mcl}$, mitral cell layer; $\mathrm{gcl}$, granule cell layer. Bar, $200 \mu \mathrm{m}$.

the glomerular layer give off more collaterals than at earlier ages. Fibers are now beginning to proliferate in the small glomeruli (Figs. $2 B, 3$ ). The fibers are similar in morphology to those observed at earlier ages.

Caudal levels of MOB are still much more heavily innervated by serotonergic fibers than are rostral levels. Fibers are especially heavy in the caudal external plexiform layer, and innervation of the glomeruli at these levels appears to be in advance of that at more rostral levels.

\section{PND 8-10}

The olfactory bulb continues to increase in diameter (1.5-2.0 $\mathrm{mm}$ in the mediolateral axis), and has also begun to elongate in the dorsoventral axis $(2.0-2.5 \mathrm{~mm})$ at this stage. Although the glomeruli are still small, averaging between 40 and $75 \mu \mathrm{m}$ in diameter, they have now become much more distinctive because they appear to be clearly surrounded by periglomerular cells. The ependymal zone begins to decrease in relative size at this and later stages (Fig. 3).

A further overall increase in the number and density of 5-HTimmunoreactive fibers is now evident (Figs. $2 C, 3,4$ ). Many fibers seem oriented parallel to the surface. The fibers often extend several hundred micrometers and cross laminar boundaries. Fibers with large terminal varicosities are still present but appear to be relatively less numerous than at earlier stages. At this age the fibers appear similar in morphology to those observed at earlier ages and the fibers in the glomerular layer are similar morphologically to those in infraglomerular layers. No consistent mediolateral differences in fiber-density patterns were observed. At this and all subsequent stages of development, the density of fibers at rostral and caudal levels of the main olfactory bulb starts becoming more uniform.

PND 14-16

The MOB continues to increase in overall size and cross-sectional area; it has enlarged substantially in the dorsal to ventral direction, to $2.0-3.0 \mathrm{~mm}$, while the mediolateral dimension is $1.5-2.0 \mathrm{~mm}$. The glomeruli are larger, averaging around $60-90$ $\mu \mathrm{m}$ in diameter, while the ependymal zone continues to narrow. The external plexiform layer has become wider $(150 \mu \mathrm{m})$, and the mitral cell layer is becoming narrower than at earlier stages.

At this stage of development, several changes in the distribution and fiber-density patterns are evident. First, the tendency for serotonergic fibers to preferentially innervate the glomerular layer is more pronounced than at earlier ages (Figs. 2D, 3-5). The density of fibers in the glomerular layer has increased to over $6 \mu \mathrm{m} / 100 \mu \mathrm{m}^{2}$. This is 3 times the density of any other layer. The density of fibers in the external plexiform layer decreases slightly, possibly because the substantial growth in this layer may have outstripped the growth of incoming serotonergic fibers at this time. The fibers in the external plexiform layer have also begun to redistribute slightly, so that most of them are located in the deeper half of the layer at this and all subsequent ages. The mitral and internal plexiform layers receive substantial input at this and later developmental stages, although the density of the fibers is about one-half the density in the glomerular layer. The estimate of total fiber length in the mitral and internal plexiform layers in each section is small (Fig. 4) because these layers occupy much less total area than the other layers of the bulb. 

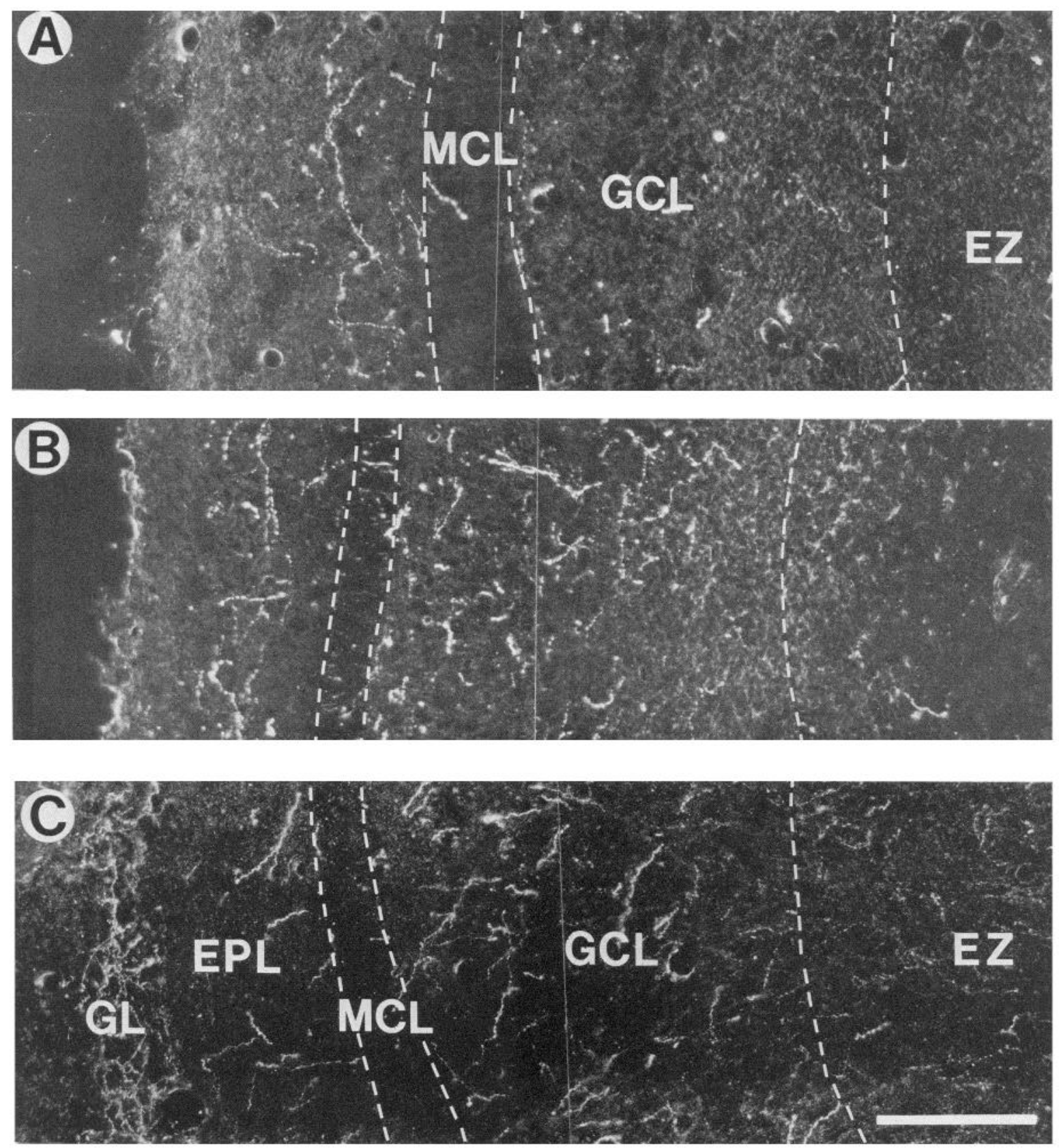

Figure 2. $A-G$, Dark-field photomicrograph montages of 5-HT-immunoreactive fibers in MOB of developing rats (transverse sections). A, PND 2. Only a few luminescent fibers are visible against a dark background. $B$, PND 4 . Fibers are now beginning to expand in glomeruli and deeper layers of MOB. $C$, PND 8. A proliferation of fibers in almost all layers has occurred at this stage. The glomeruli $(G L)$ have become heavily innervated by 5 -HT at this earlier age. $D$, PND 15 . Most layers show an increase in fiber label at this time. The internal plexiform layer $(I P L)$ has now become identifiable. $E$, PND 21. Continued increase in 5-HT fibers is evident at this time. $F$, PND 39 . The glomeruli have increased considerably in size and there are abundant fibers present in this and other olfactory laminae. $G$, Adult. By PND 64 and older, fibers are still very dense in the glomerular layer. Fibers are clearly present in other laminae, although their density is much less than those in the glomerular layer. $G L$, glomerulus; $E P L$, external plexiform layer; $M C L$, mitral cell layer; $I P L$, internal plexiform layer; $G C L$, granule cell layer; $E Z$, ependymal zone. Bar, $100 \mu \mathrm{m}(A-G)$.

The density of fibers in all layers, except for the glomerular layer, has peaked by this time period. At all subsequent ages, the fiber density in the infraglomerular layers remains relatively constant. By contrast, the fiber density of 5-HT fibers in the glomerular layer continues to increase steadily from this age into adulthood. A trend that has become evident by this time is for the 5-HT fibers to become segregated into 2 morphological types: the fibers in the glomeruli, which are thicker and contain larger varicosities than the fibers in infraglomerular layers. In all layers, the average intervaricosity distance has increased. The intervaricosity interval now ranges from 1 to $15 \mu \mathrm{m}$, as opposed to $1-7 \mu \mathrm{m}$ at earlier stages.

\section{PND 21-43}

The MOB continues to increase in size, especially in the dorsal to ventral direction. Thus, the bulb has become elongated to around $3.5 \mathrm{~mm}$ dorsal to ventral, while the width is $1.5-2.0$ $\mathrm{mm}$. The glomeruli, external plexiform layer, and granule cell 

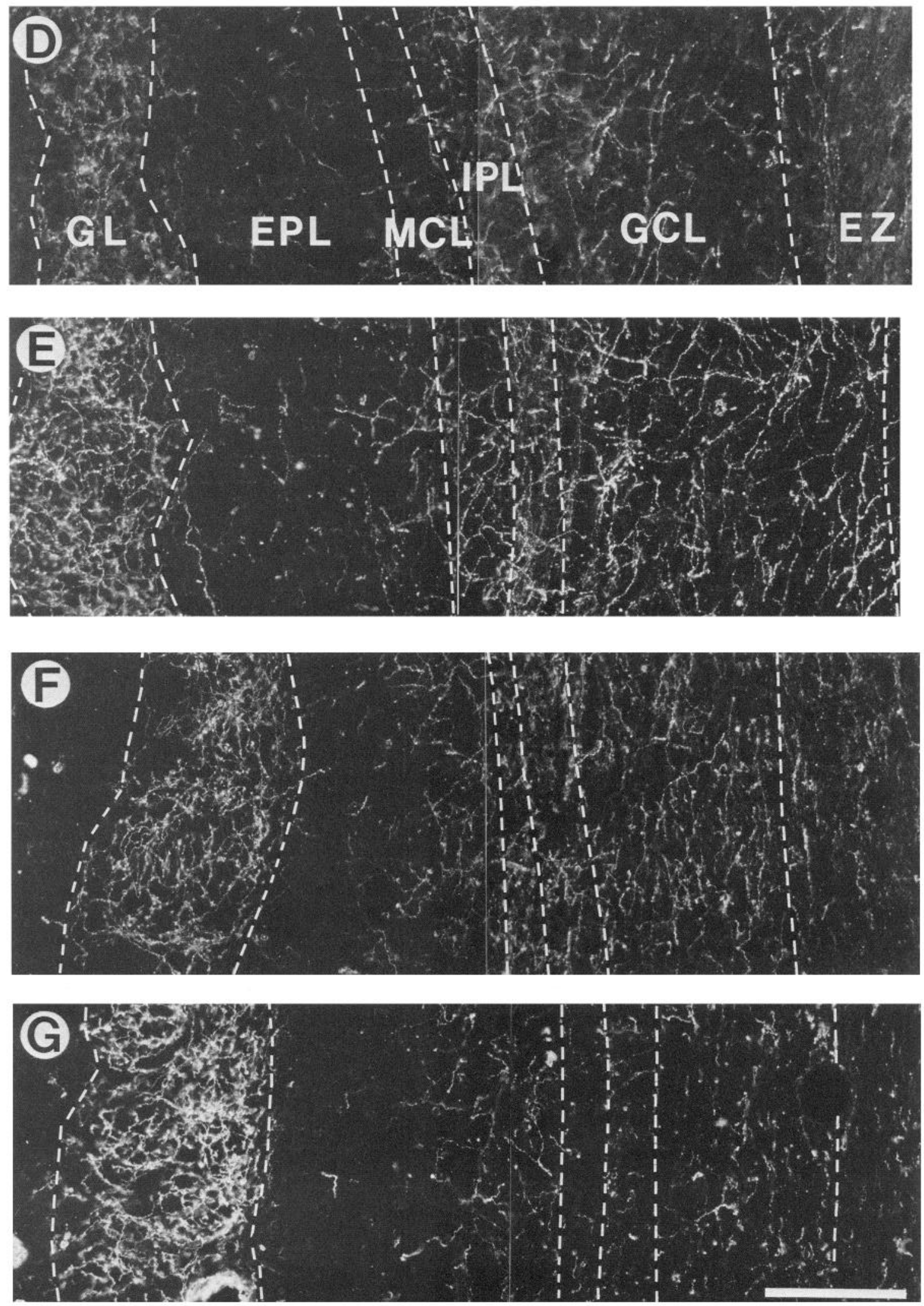

Figure 2. Continued.

layers have all increased in size. The glomeruli range from 45 to $120 \mu \mathrm{m}$ in diameter, the external plexiform layer is $100-250$ $\mu \mathrm{m}$ wide, and the granule cell layer is approximately $400 \mu \mathrm{m}$ wide. By visual inspection, the density of fibers in the glomeruli appears to still be increasing, while the fibers in other layers are holding steady (Figs. 2, $E, F ; 3$ ). Morphometric analysis confirmed this visual impression. The fiber density in the glomeruli has increased to $7-8 \mu \mathrm{m} / 100 \mu \mathrm{m}^{2}$, while that in other layers 

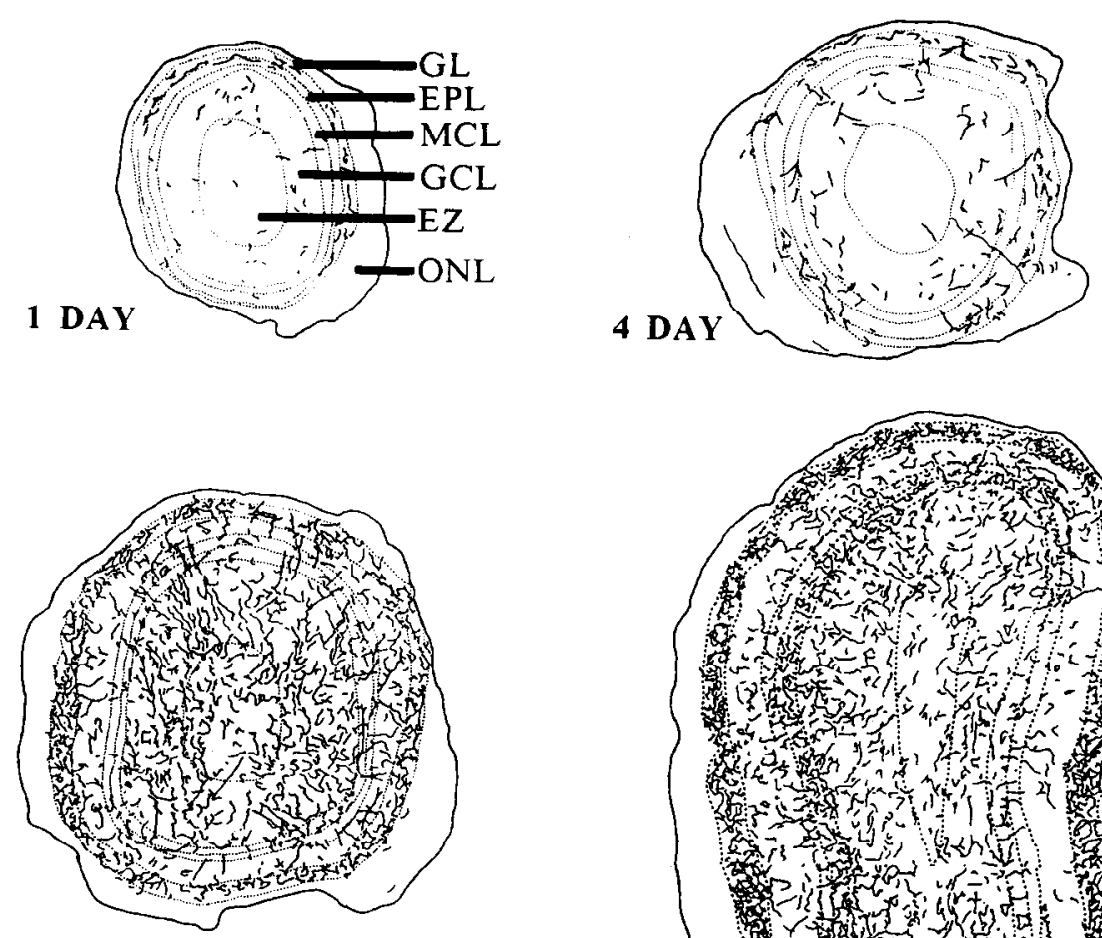

8 DAY

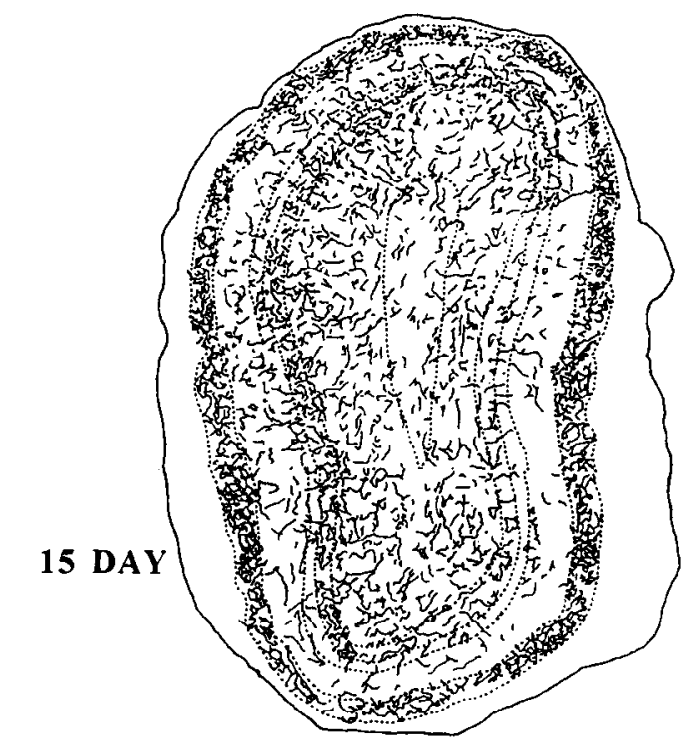

Figure 3. Computer-assisted drawings of developing rat MOB at PND 1 , $4,8,15,39$, and in the adult. Bar, 1 $\mathrm{mm}$. Abbreviations, see Figure 2. $O N L$, Olfactory nerve layer.

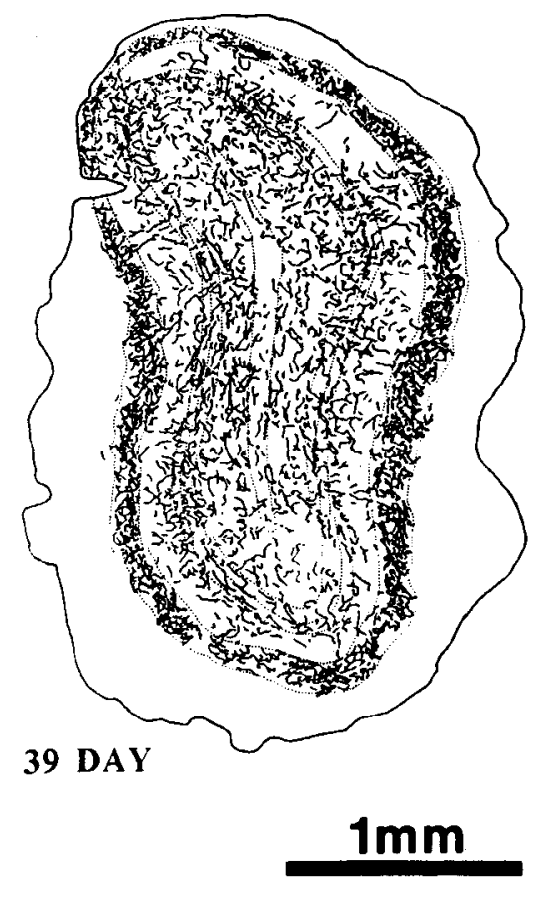



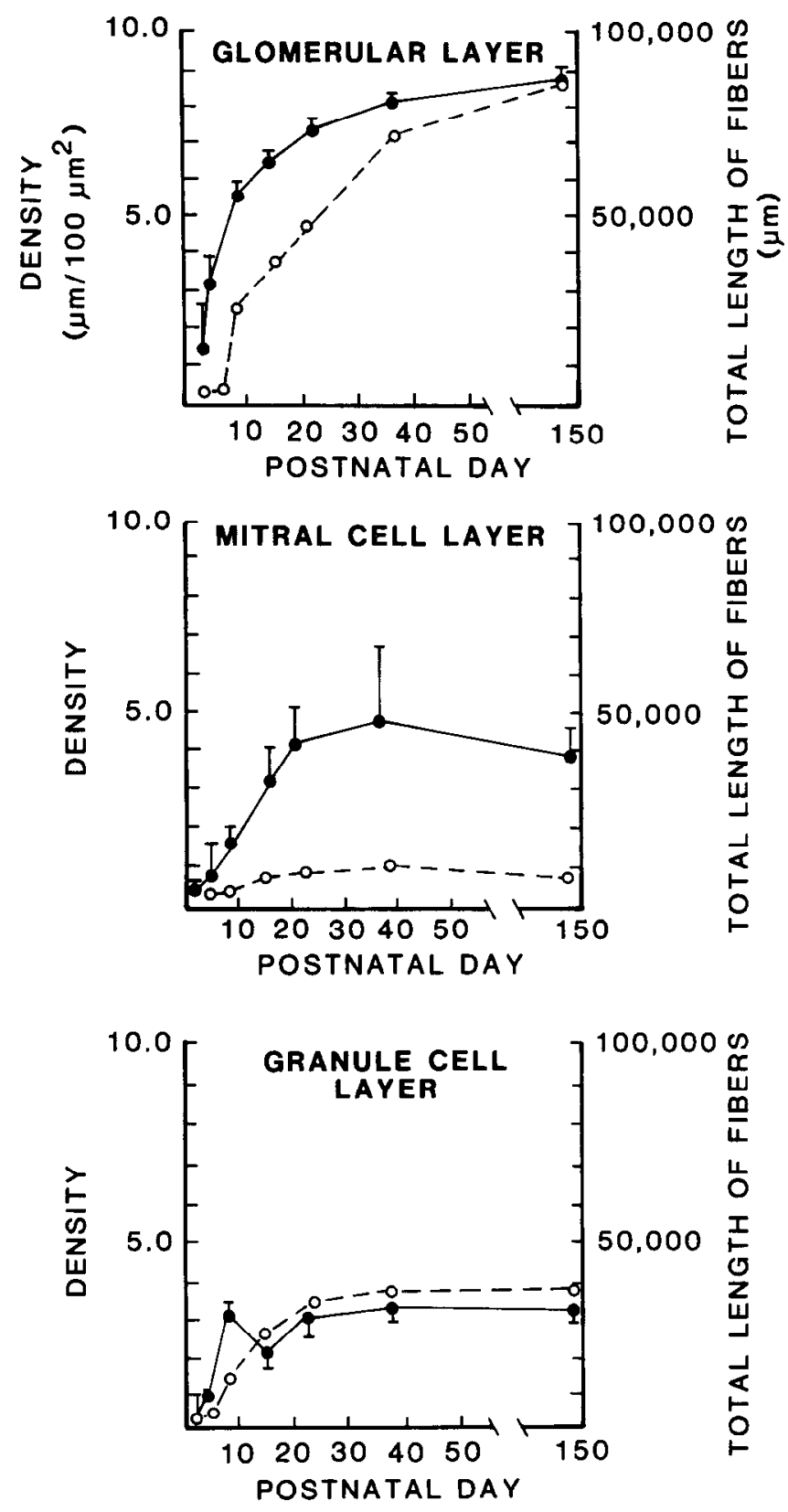
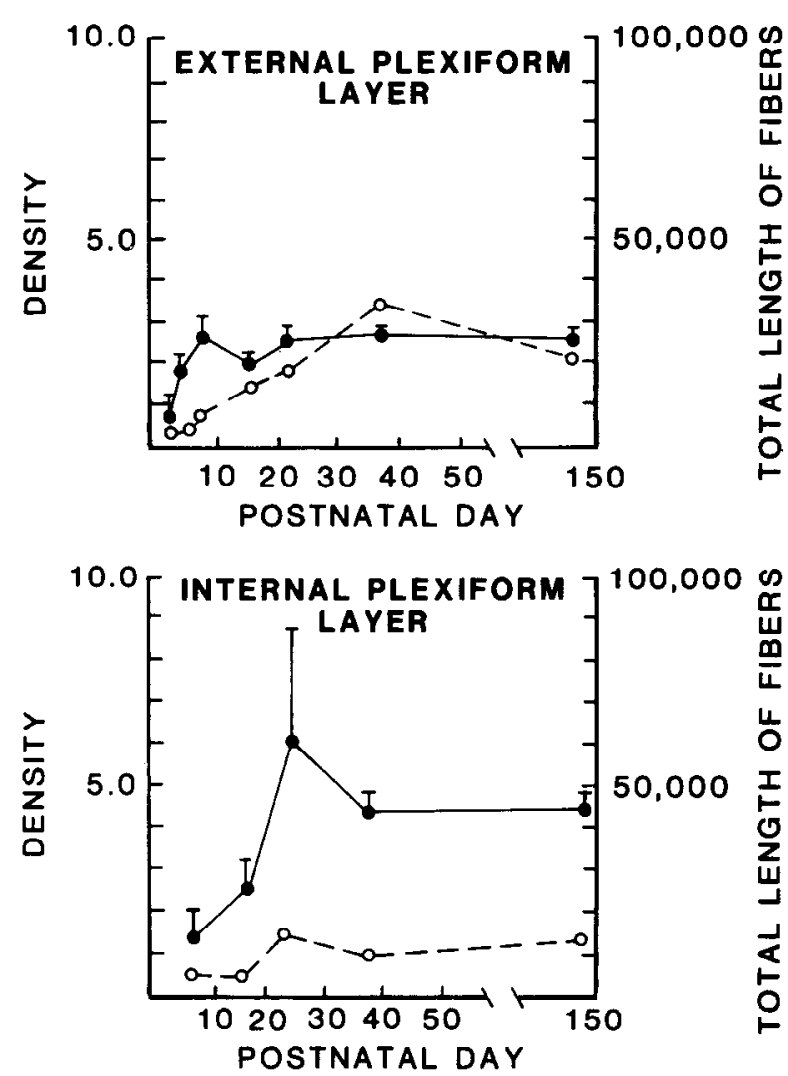

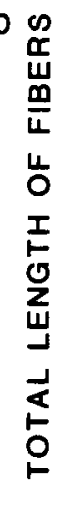

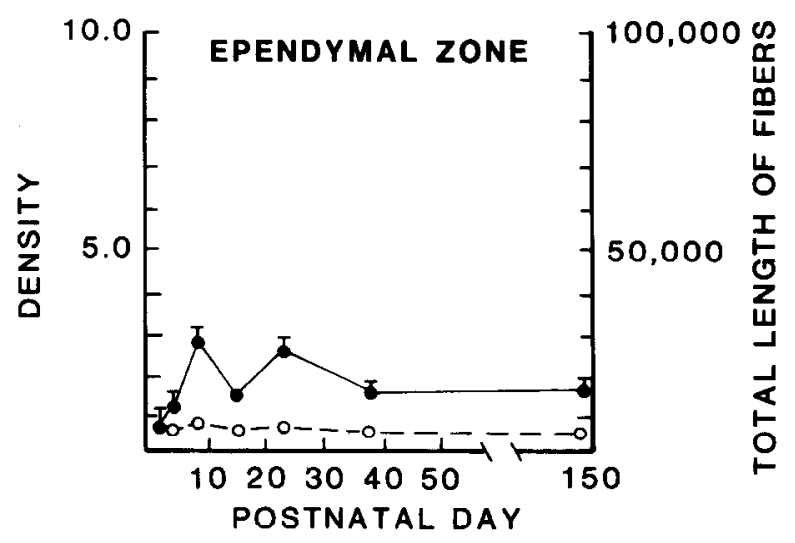

$\longrightarrow$ Density of Fibers/Unit Area

O- - Estimated Total Length of Fibers/30 $\mu \mathrm{m}$ Section

Figure 4. Graph depicting trends in 5-HT fiber deployment in different layers of MOB during development. Each plotted point for the density measurements represents the mean and SD ( $n=3-4$ rats). The plotted curve for estimated total length of fibers in each section is an extrapolation derived from density and cross-sectional area measurements in each lamina.

remains relatively unchanged (Figs. 4, 5). The mitral and internal plexiform layers have a relatively heavy input of 5-HT fibers, just as at earlier ages, but the layers remain thin so that the total fiber length in these layers is still low. Thus, although the density of input is around $4-5 \mu \mathrm{m} / 100 \mu \mathrm{m}^{2}$, relatively few fibers contribute to these thin layers.

Adult (60 $d$ and older)

In the adult rat, the MOB is $3-4 \mathrm{~mm}$ between the dorsal and ventral borders, and the width is $1.5-2.0 \mathrm{~mm}$. Thus, the MOB has continued to enlarge in the dorsoventral direction although the width of the bulb has remained constant from the previous stage.

The density of serotonergic fibers fluctuates in the adult MOB more than in any other period. The fluctuation appears to be due to differences in fixation procedures. The fibers that are most susceptible to subtle experiment-to-experiment variations in fixation are the thin, lightly stained fibers that are predominantly found in infraglomerular layers.

Two distinct types of fibers are present in the adult MOB. 


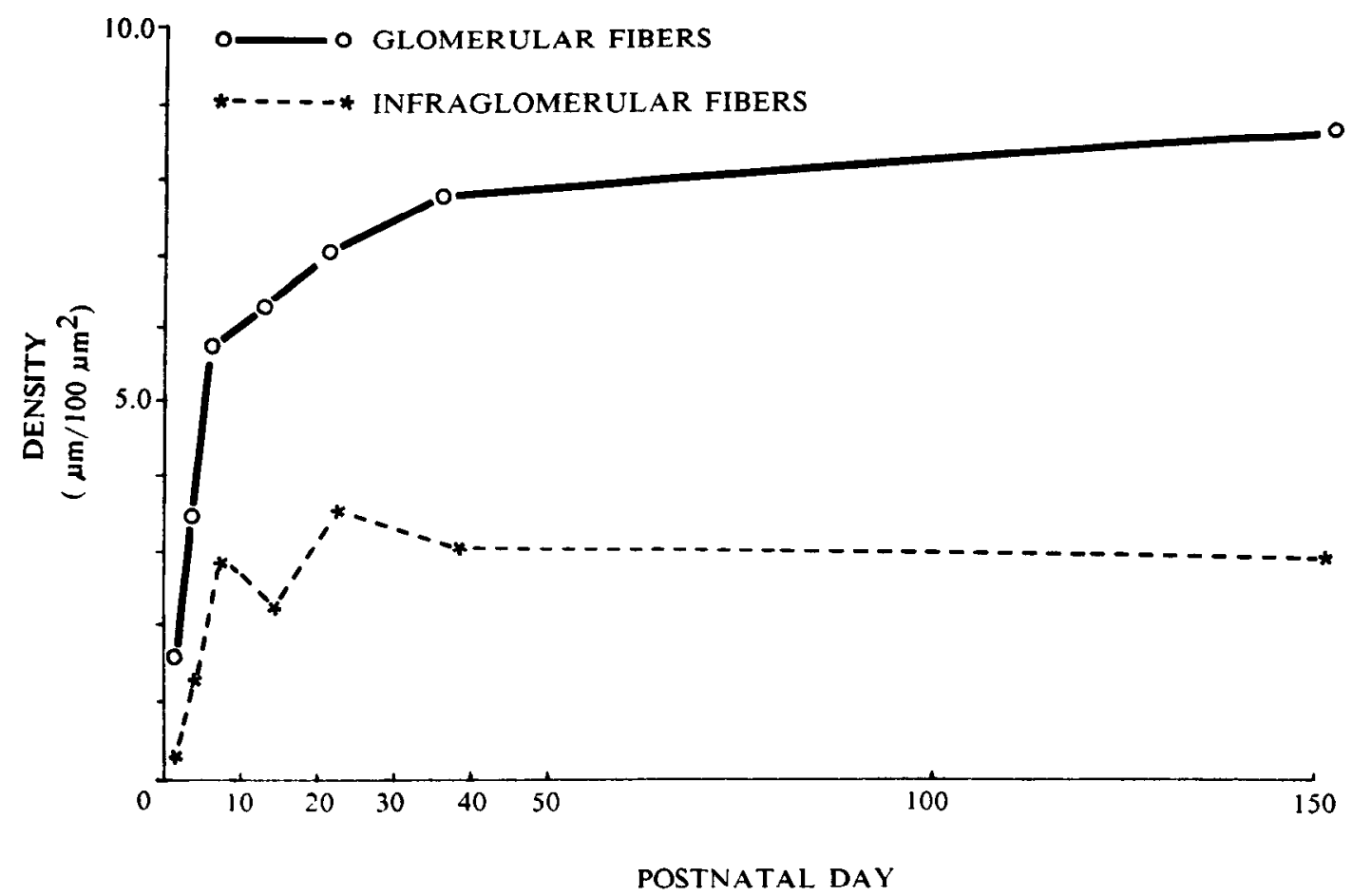

Figure 5. Graph showing the density of 5-HT-immunoreactive fibers in the glomerular layer compared to the combined average density in infraglomerular layers.

Thick, intensely stained fibers, approximately $0.33 \mu \mathrm{m}$ in diameter with varicosities averaging $1 \mu \mathrm{m}$ in diameter, predominate in the glomeruli. Fibers of this kind are also present in infraglomerular layers, especially the external plexiform layer, but they contain smaller varicosities, which average just over $0.5 \mu \mathrm{m}$. The second fiber type, consisting of very fine axons that average $0.25 \mu \mathrm{m}$ in diameter, is the predominant element in the infraglomerular layers. These fibers are generally lightly stained and contain small varicosities that are barely larger than the diameter of the fiber itself. The distance between varicosities varies between 1 and $30 \mu \mathrm{m}$ in both glomerular and infraglomerular axons so that there is no consistent intervaricose distance that might help to further differentiate the fiber types.

The laminar densities of 5-HT fibers in the adult MOB have been described in the preceding paper (McLean and Shipley, 1987). The glomerular layer continues to be the major recipient of 5-HT innervation relative to other laminae (Figs. 4, 5). The glomerular layer contains approximately 3 times the density of 5-HT fibers of the infraglomerular layers (Fig. 5) and, as noted, these fibers are predominantly thick, with large varicosities. Estimates of total 5-HT fiber length ( $30 \mu \mathrm{m}$ section) for each layer indicate that the glomerular layer has at least 3 times the total length of 5-HT fibers of the second densest layer, the granule cell layer (Fig. 4).

Compared to other developmental ages, the adult MOB shows a stabilized density of 5-HT-immunoreactive fibers in all laminae except for the glomerular layer, where there continues to be a slight increase in fiber density (Figs. 4, 5). This stabilization of infraglomerular 5-HT fiber density is evident from approximately the third postnatal week. Since the olfactory bulb continues to increase in size from birth to maturity, and because the density of 5-HT fibers remains fairly constant after the second postnatal week, it would appear that 5-HT fibers must continue to grow in length into adulthood.

\section{Anterograde WGA-HRP versus 5-HT-immunoreactive staining}

To assess whether serotonin is expressed at sufficient levels to be detected in MOB fibers by our immunocytochemical methods, we compared 5-HT-immunoreactive fiber distribution to the distribution of anterograde labeling in $\mathrm{MOB}$ following injections of WGA-HRP in the dorsal and median raphe nuclei in PND-14 animals. The distribution of anterograde label closely resembled the pattern of 5-HT label in MOB at this relatively immature developmental age (Fig. 6). This suggests that, at this age at least, fibers that have reached MOB from the raphe probably express immunocytochemically detectable levels of 5-HT, and thus that the immunocytochemical staining method provided an accurate picture of the distribution and density of 5-HT raphe fibers in the bulb during development.

\section{Discussion}

This study has demonstrated that the serotonergic innervation of the rat olfactory bulb is essentially a postnatal event. There are distinctive distributions of 5-HT-immunoreactive fibers in the rat $\mathrm{MOB}$ at different stages of development. Innervation begins with a relatively nonspecific distribution and becomes highly laminated with maturation. At birth, there are few 5-HT fibers in the bulb, but during the first postnatal week there is a sharp increase in the number and density of fibers in all layers of the bulb. During the first postnatal week, 5-HT fibers are much denser and more extensively ramified in the caudal parts of MOB. In the second week, the fibers continue to proliferate, especially in the glomerular layer. Following the third week, the 5-HT fiber density in the infraglomerular layers begins to gradually level off. The density of 5-HT fibers in the glomerular layer continues to increase gradually into adulthood. Since all layers of the olfactory bulb continue to expand into adulthood (Meis- 

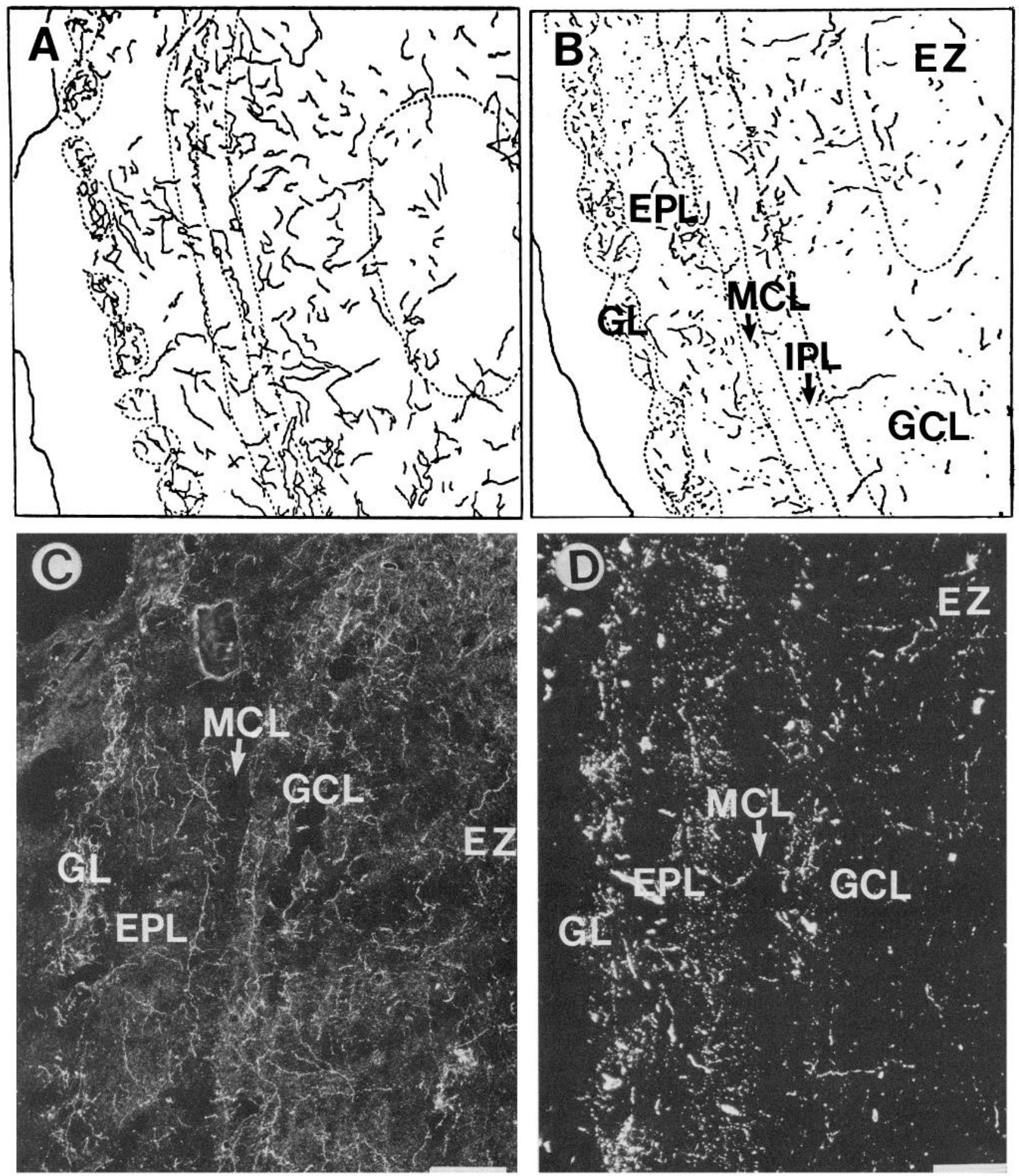

Figure 6. Computer-assisted drawings $(A, B)$ of dark-field photomicrographs $(C, D)$, respectively, of MOB section of PND 14 rat. There are striking similarities in label pattern between 5-HT-immunoreactive fibers $(A, C)$ and anterograde label $(B, D)$ following injection of WGA-HRP into raphe nuclei at this age; all layers have substantial 5-HT fiber staining. The anterograde label, although less robust than the 5-HT immunoreactivity, is similarly distributed to the 5-HT. Bar, $100 \mu \mathrm{m}(C, D)$; same magnification in $A$ and $D$. Arrows demarcate the thin MCL and IPL.

ami, 1979), 5-HT fibers must be growing continuously in order to maintain the steady levels of 5-HT fiber density observed from the third postnatal week.

The early preferential innervation of 5-HT fibers in the caudal part of the bulb suggests that this centrifugal afferent system could be involved in the preferentially high levels of 2-deoxyglucose activity reported in the caudal bulb in the neonatal rat (Teicher et al., 1980; Greer et al., 1982). Thus, the heightened metabolic activity in the region of the "modified glomerular complex" of young animals may reflect the activation of brain- 
stem afferents that arborize preferentially in the caudal parts of the developing olfactory bulb. Although little is known about the precise function of these generalized subcortical projection systems, it seems likely that their activation might be associated more with general aspects of sensory stimulation (e.g., arousal) than with specific features of sensory stimuli. Therefore, it is possible that the preferentially high metabolic activity in the caudal part of the perinatal rat olfactory bulb might reflect the differential maturation of 5-HT and possibly other developing subcortical afferents more than the selective activation of specific nasal epithelial receptor populations by odorant molecules.

\section{Fiher deployment}

A technical consideration must be addressed at this point. In our initial experiments it appeared that our immunocytochemical labeling was not as strong as in cases following modification to the perfusion protocols. The initial results differed from those of later experiments in that far fewer infraglomerular fibers were detected in MOB of older animals. These results led us to speculate that 5-HT fibers decrease in infraglomerular layers in the adult (McLean et al., 1985). Subsequent experiments proved otherwise; serotonergic fibers were maintained in infraglomerular layers into adulthood, albeit much less densely than in the glomerular layer. The fibers that were not consistently observed in our initial experiments belong to a class of axons that are thin, weakly stained, and susceptible to modification of perfusion protocol (see Results, and McLean and Shipley, 1987). There may still be, however, a laminar redistribution of 5-HT fibers during development.

Recent studies of developing fibers indicate that fibers that fail to find proper synaptic connections are retracted (Innocenti et al., 1977; Kandel, 1985). In the olfactory bulb, the density of serotonergic fibers does not decrease during development, suggesting that there is no net loss of these fibers in any layer of this cortical structure. However, we cannot exclude the possibility that some serotonergic fibers retract and are replaced by others, so that the overall density of fibers is maintained. The segregation of fibers in different layers (mainly thick fibers in the glomerular layer and thinner fibers in the infraglomerular layers) in the adult animals suggests that there may be a sortingout of fiber types. We have not yet attempted to analyze the developmental fate of thick versus thin fibers.

Recent evidence has indicated that, in some systems, presynaptic fibers comigrate with newly proliferated postsynaptic target ncurons in the developing chick brain stem (Young and Rubel, 1986). The ingrowth and arborization of 5-HT-immunoreactive fibers coincide with a period of considerable neuronal proliferation and migration in the developing olfactory bulb. Thus, it is possible that the 5-HT fibers make initial synaptic contact with their target neurons while these neurons are still migrating to their final destination in the olfactory bulb. When the neurons reach their definitive location, the afferent 5-HT fibers might arborize as the processes of target cells mature. The increasing ramification of serotonergic fibers in the glomerular layer throughout most of the postnatal development period is consistent with this possibility. The dendrites of juxtaglomerular cells ramify extensively in the glomeruli and are likely targets of 5-HT fibers (Halasz et al., 1978). These cells are born in the ependymal zone and migrate to their glomerular position postnatally (Bayer, 1983). Juxtaglomerular neurons are still being generated in the ependymal zone into the second postnatal week.
This is the same time that 5-HT fibers are abundant in the infraglomerular layers. These migrating juxtaglomerular cells may constitute the appropriate target for the arriving 5-HT fibers. The thin infraglomerular 5-HT fibers may remain in the infraglomerular layers because their targets remain in these layers. A likely postsynaptic target of the infraglomerular 5-HT fibers is the granule cell population. Most of these cells are born postnatally (Bayer, 1983) and remain deep to the mitral cell layer. Their dendrites extend radially to the external plexiform layer or towards the ependymal zone. Thus, serotonergic fibers may contact and co-arborize with the differentiating dendrites of granule cells and remain in the infraglomerular location.

\section{Functional roles of 5-HT fibers during development}

Previous investigators have suggested that serotonin may have developmental functions other than as a neurotransmitter. For instance, Lauder et al. (1982) have suggested that 5-HT fibers may act by influencing the timing of cell migration. The present study does not address this question directly, but some of our findings bear on this issue. If 5-HT fibers have some extratransmissional role during development, then one would expect the fibers to be present and to contain 5-HT during that period. Studies using ${ }^{3} \mathrm{H}$-thymidine have shown that most of the extrinsic projection neurons of MOB (mitral and internal tufted cells) are born prenatally and thus prior to the arrival of all but a fraction of the 5-HT fibers (Altman, 1969; Bayer, 1983). However, most of the interneurons in $\mathrm{MOB}$ are born during the first 3 postnatal weeks, and a significant fraction of bulb interneurons, including juxtaglomerular cclls, are born aftcr PND 20 (Altman, 1969; Bayer, 1983). Thus, the differentiation and migration of olfactory interneurons correlates very well with the period of arrival and proliferation of 5-HT fibers in MOB. During this protracted postnatal period of neuronal migration and differentiation, 5-HT fibers could have an influence on a significant proportion of interneurons in MOB but probably not on the output neurons that are born and differentiate earlier in development. Thus, our results would suggest that if 5-HT fibers have an epigenetic role, this role is probably targeted on specific populations of MOB interneurons.

An alternative role of 5-HT in development has been proposed by Lidov and Molliver (1982). They found that 5-HT fibers did not arborize significantly in regions of cerebral cortex that were still undergoing cellular proliferation; rather, 5-HT fibers appeared to arborize mainly after the presumed target neurons had reached a certain stage of maturity. Thus, these investigators suggested that the serotonin may function to inhibit or terminate the period of cortical circuit refinement during development. Unfortunately, the specific neuronal targets of 5-HT fibers in the neocortex are unknown, and it is also not known whether all the neuronal types that eventually comprise a cortical layer are born at the same time. It is not clear, then, whether there is any obvious relationship between the period when 5-HT fibers enter the cortex and arborize in their definitive layer and the time of migration and/or differentiation of cortical neurons that are targets of 5-HT fibers.

Another interesting recent study has correlated the development of endogenous 5-HT levels and 5-HT receptors to "critical periods" in cat visual cortex development (Jonsson and Kasamatsu, 1983). The results indicated that 5-HT could play a role in triggering the onset of critical period plasticity, but not in maintaining the critical period. The relationship between $5-\mathrm{HT}$ 
levels and 5-HT receptors in the developing olfactory bulb is not known, but studies of this kind may further elucidate the potential role(s) of 5-HT in development.

One final line of evidence suggesting a developmental role for serotonin has come from tissue culture studies. Haydon et al. (1984) demonstrated that serotonin inhibits neurite outgrowth in cultured neurons. If 5-HT has a similar action in vivo, then the serotonergic fibers could function to terminate, inhibit, or perhaps "sculpture" the formation of synaptic contacts in MOB. This kind of regulatory role is particularly interesting to consider with regard to the olfactory bulb glomeruli because there is probably a large amount of synaptic turnover in the glomeruli throughout the life of the animal. The major synaptic input to glomeruli is the terminals of the olfactory nerve. The cell bodies giving rise to the olfactory nerve are the primary olfactory neurons (PONs) of the nasal epithelium. It is well established that there is continuous turnover of PONs that differentiate from olfactory epithelial basal cells. New PONs form new synaptic contacts in the glomeruli of MOB. Thus, the glomeruli represent a site where there is some degree of continual synaptic turnover. If 5-HT regulates neurite extension during development, then it is entirely conceivable that it continues to do so in response to glomerular synaptic turnover throughout the animal's life. In this regard, it is interesting that 5-HT fibers in the glomeruli are markedly different from those in the infraglomerular layers. Glomerular 5-HT fibers are thick, bear large varicosities, and are intensely stained by 5-HT immunocytochemistry. It may also be significant that 5-HT fiber density appears to still be increasing slightly even in the oldest animals studied.

In summary, we have found that serotonergic innervation of the main olfactory bulb occurs almost entirely after birth. During the first 2-3 postnatal weeks the fibers proliferate in all olfactory bulb layers. The glomerular layer is the predominant recipient of 5-HT fibers soon after the fibers arrive in the MOB and throughout development. Subsequent to the third postnatal week, the increased density of 5-HT fiber proliferation subsides, so that a steady state of 5-HT fiber density remains in most MOB laminae. Several possible functions of serotonin in development have been postulated by others. Given the coincident time periods of interneuronal propagation/migration (Bayer, 1983) and the arrival and arborization of serotonergic fibers in $\mathrm{MOB}$, it is conceivable that serotonergic fibers have developmental regulatory functions in MOB; serotonergic fibers could have a role in regulating cell migration (Lauder et al., 1982), in inhibiting "plasticity" (Lidov and Molliver, 1982) or in regulating synaptic contacts (Haydon et al., 1984). Further experiments are needed to test these potential developmental functions of 5-HT fibers. The MOB should provide a model for such studies.

\section{References}

Altman, J. (1969) Autoradiographic and histological studies of postnatal neurogenesis. IV. Cell proliferation and migration in the anterior forebrain, with special reference to persisting neurogenesis in the olfactory bulb. J. Comp. Neurol. 137: 433-458.

Basbaum, A. I., and H. L. Fields (1979) The origin of descending pathways in the dorsolateral funiculus of the spinal cord of the cat and rat: Further studies on the anatomy of pain modulation. J. Comp. Neurol. 187: 209-224.

Bayer, S. A. (1983) ${ }^{3} \mathrm{H}$-thymidine-radiographic studies of neurogenesis in the rat olfactory bulb. Exp. Brain Res. 50: 329-340.
Feldberg, W., and R. D. Myers (1964) Effects on temperature of 5-hydroxytryptamine, adrenaline, and noradrenaline injected into the cerebral ventricles or the hypothalamus of cats. J. Physiol. (Lond.) 173: $25 \mathrm{P}$.

Greer, C. A., W. B. Stewart, M. H. Teicher, and G. M. Shepherd (1982) Functional development of the olfactory bulb and a unique glomerular complex in the neonatal rat. J. Neurosci. 2: 1744-1759.

Halasz, N., A. Ljungdahl, and T. Hökfelt (1978) Transmitter histochemistry of the rat olfactory bulb. II. Fluorescence histochemical, autoradiographic and electron microscopic localization of monoamines. Brain Res. 154: 253-271.

Haydon, P. G., D. P. McCabb, and S. B. Kater (1984) Serotonin selectively inhibits growth cone mobility and synaptogenesis of specific identified neurons. Science 226: 561-564.

Innocenti, G. M., L. Fiore, and R. Caminiti (1977) Exuberant projection into the corpus callosum from the visual cortex of newborn cats. Neurosci. Lett. 4: 237-242.

Jonsson, G., and T. Kasamatsu (1983) Maturation of monoamine neurotransmitters and receptors in cat occipital cortex during critical period. Exp. Brain Res. 50: 449-458.

Jouvet, M. (1962) Recherches sur les strictures nerveuses et les mecanismes responsables des differentes phases du sommeil physiologiques. Arch. Ital. Biol. 100: 125-206.

Kandel, E. R. (1985) Synapse formation, trophic interactions between neurons, and the development of behavior. In Principles of Neural Science, E. R. Kandel and J. H. Schwartz, eds., pp. 743-755, Elsevier, New York.

Lauder, J. M., J. H. Wallace, H. Krebs, P. Petrusz, and K. McCarthy (1982) In vivo and in vitro development of serotonergic neurons. Brain Res. Bull. 9: 605-625.

Lidov, H. G. W., and M. E. Molliver (1982) An immunohistochemical study of serotonin neuron development in the rat: Ascending pathways and terminal fields. Brain Res. Bull. 8: 389-430.

McLean, J. H., and M. T. Shipley (1987) Serotonergic afferents to the rat olfactory bulb: I. Origins and laminar specificity of serotonergic inputs in the adult rat. J. Neurosci. 7: 3016-3028.

McLean, J. H., M. Lazoff, E. B. Sieloff, W. T. Nickell, and M. T. Shipley (1985) The ontogenic development of serotonergic fibers in the rat olfactory bulb. Soc. Neurosci. Abstr. 11: 65.

Meisami, E. (1979) The developing rat olfactory bulb: Prospects of a new model system in developmental neurobiology. In Neural Growth and Differentiation, E. Meisami and M. A. Brazier, eds., pp. 183206, Raven, New York.

Mesulam, M.-M. (1978) Tetramethyl benzidine for horseradish peroxidase neurochemistry: A non-carcinogenic blue reaction-product with superior sensitivity to visualizing neural afferents and efferents. J. Histochem. Cytochem. 26: 106-117.

Mizuno, H., P. K. Talwalker, and J. Meites (1967) Central inhibition by serotonin of reflex release of oxytocin on response to suckling stimulus in the rat. Neuroendrocrinology 2: 222-231.

Myers, R. D., A. Kawa, and D. B. Beleslin (1969) Evoked release of 5-HT and NEFA from the hypothalamus of the conscious monkey during thermoregulation. Experientia 25: 705-706.

Shipley, M. T. (1982) Insular cortex projection to the nucleus of the solitary tract and brainstem visceromotor regions in the mouse. Brain Res. Bull. 8: 139-148.

Srebro, B., and S. A. Lorens (1975) Behavioral effects of selective midbrain raphe lesions in the rat. Brain Res. 89: 303-325.

Teicher, M. H., W. B. Stewart, J. S. Kauer, and G. M. Shepherd (1980) Suckling pheromone stimulation of a modified glomerular region in the developing rat olfactory bulb revealed by the 2-deoxyglucose method. Brain Res. 194: 530-535.

Tenen, S. S. (1968) Antagonism of the analgesic effect of morphinc and other drugs by $p$-chlorophenylalanine, a serotonin depletor. Psychopharmacologia 12: 278-285.

Vogt, M. (1974) The effect of lowering the 5-hydroxytryptamine content of the rat spinal cord on analgesia produced by morphine. J. Physiol. (Lond.) 236: 483-498.

Young, S. R., and E. W. Rubel (1986) Embryogenesis of arborization pattern and topography of individual axons in $n$. laminaris of the chicken brain stem. J. Comp. Neurol. 254: 425-459. 